\title{
Executive Director's Report
}

C reating greater media awareness of Canadian forests and our forestry practices is a key initiative of the Institute. Being proactive in informing the media and the public about forests and forestry is one part, providing scientific, factual information, and being accessible is another component of communication.

The Institute has twenty three sections across the country. Our section directors and chairs have been provided with material for communicating with the media, and have been asked to identify communication officers and media contacts. The sections have been encouraged to be proactive in working with the media and public and have been asked to report media responses, so we may provide national coverage on events. Every Institute member in their own capacity has the ability with- in their own area of expertise to speak on forestry and/or forestry-related issues as a practitioner and to provide correct information to the public and media. I would encourage members to become involved and talk with their section councils on media events that can be organized, or identify topics that should be discussed in your region.

The Canadian Institute of Forestry/ Institut forestier du Canada has a series of position statements and policy papers. A new redesigned compendium of these papers is posted on the Institute's website (www.cif-ifc.org) and the papers are also available in hard copy from National office. As a source for additional information, the Institute's website has information on Canadian forest practices and forests in Canada.

I have found that local community newspapers appreciate receiving infor-
Roxanne Comeau R.P.F. CIF/IFC Executive Director/ Directrice exécutive

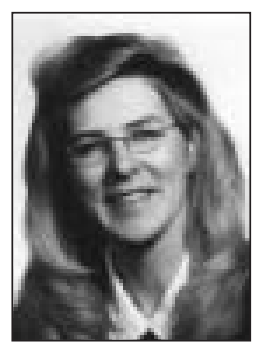

mation on community events. I would encouragemembers to send an announcement with photos of a community activity such as tree planting, provide information about privateland forestry and maple syrup production, or in the fall - why leaves change colours, in addition to responding to regional issues and topics of concern. If your section has a website, encourage your section to provide information on forests in your region so as to direct media inquires to local forestry information.

If there is a topic or issue that you would like the Institute to be involved in, please send mean email : rcomeau@ cif-ifc.org. O ur Executivewill review the topic or issue, and will discuss action, if appropriate. Section councils will also be involved in making decisions on actions.

\section{Rapport de la directrice exécutive}

A ccroître la sensibilisation des médias relativement aux forêts du Canada et à nos pratiques forestières constitue l'une des principales initiatives de l'Institut. Êtreen mesure d'informer les médias et le public sur ce qui toucheles forêts et la foresterie constitue une facette, alors que l'apport d'information scientifique et factuelle tout en étant accessible en constitue l'autre.

L'Institut compte 23 sections réparties dans tout le pays. Les directeurs et les présidents de section ont reçu de notre part du matériel pour communiquer avec les médias et nous leur avons demandé d'identifier des agents de communication et des personnesressources pour les médias. Les sections ont été incitées à être proactives dans leurs relations avec les médias et le public et nous leur avons demandé de faire état des réactions des médias de telle sorte qui nous puissions offrir une couverture nationale lorsque requis. Chaque membre de l'Institut, dans la mesure de ses moyens, a la possibilité dans son domaine de savoir-faire de parler de foresterie ou de sujets reliés à la foresterie en tant que professionnel et de transmettre de l'information adéquate. J'inciterais les membres à participer et à discuter avec leur conseil de section des activités médiatiques à organiser ou pour identifier les sujets intéressants dans votre région.

Le Canadian Institute of Forestry/ Institut forestier du Canada a réuni une série d'énoncés et de propositions. Une récente mise à jour d'un recueil de ces documents est affichée sur le site Internet de l'Institut (www.cif-ifc.org) et ces documents sont également disponibles sous forme de publication en s'adressant au bureau national. Le site Internet del'Institut comporte d'autres renseignements sur les pratiques forestières au Canada et sur les forêts canadiennes.

J'ai constatéqueles journaux locaux aiment recevoir de l'information reliée aux activités communautaires. J'inciterais les membres à acheminer les textes publicitaires accompagnés de photos dans le cadre d'activités comme une plantation d'arbres, à transmettre de l'information sur la foresterie en terrain privé et la production de sucre d'érable ou, encore, au cours de l'automneà savoir pourquoi les feuilles changent de couleur, en plus de répondre aux enjeux et sujets d'intérêt régionaux. Si votre section utilise un site Internet, incitez votre section à fournir de l'information sur les forêts de votre région de manière à diriger les demandes des médias vers de l'information locale en matière de foresterie.

Si vous souhaitez que l'Institut s;engage sur un sujet ou un enjeu, veuillez me faire parvenir un courriel à : rcomeau@cif-ifc.org. Notre conseil exécutif étudiera le sujet ou l'enjeu et décidera des suites à donner. Les conseils de section participeront également à la prise de décision. 


\section{Canadian Institute of Forestry/Institut forestier du Canada}

\section{Suite 606, 151 Slater St., Ottawa, Ontario, Canada K1P 5H3 Tel./Tél: (613) 234-2242 Fax/Téléc: (613) 234-6181 E-mail/courrier électronique: cif@cif-ifc.org Website/site Internet: www.cif-ifc.org}

The Canadian Institute of Forestry/Institut forestier du Canada ( $\mathrm{CIF/IFC)} \mathrm{has} \mathrm{been} \mathrm{the} \mathrm{national} \mathrm{voice} \mathrm{for} \mathrm{forestry} \mathrm{professionals} \mathrm{since}$ 1908. Our membership includes foresters, forest technologists and technicians, educators, scientists and others with a professional interest in forestry. We work in government, industry, research, education and consulting and represent one of the largest professional voices for forestry in Canada.

Our Mission The M ission of the CIF/IFC is to advance the stewardship of Canada's forest resources, provide national leadership in forestry, promote competence among forestry professionals, and foster public awareness of Canadian and international forestry issues.

Our Values and Beliefs We believethat members of the Canadian Institute of Forestry/Institut forestier du Canada (CIF/IFC) should have an intimate knowledge and understanding of Canada's forests. We believe that the stewardship of our forests must be based on an understanding of ecological principles coupled with appropriate inventory and monitoring to ensure that the criteria of ecological integrity and function are maintained within an acceptable sociological framework. Through this understanding, members should promote the intelligent use of Canada's forests to provide a sustainable flow of a wide range of forest commodities, representing a balance of economic, spiritual, recreational and wildlife habitat values. A wide range of forest alternatives is possible, ranging from natural forests to intensively managed tree farms. We believe that the mix of forest uses required to satisfy Canadian needs should be based on an informed public dialogue. Our members should play a constructive role in facilitating such debate.

L'Institut est le porte parole des professionnels de la foresterie depuis 1908. Nos membres regroupent des forestiers, des techniciens forestiers, des enseignants, des chercheurs et d'autres personnes qui ont un intérêt professionnel pour la foresterie. Nous travaillons pour le gouvernement, l'industrie, en recherche, en éducation et en consultation et nous sommes l'un des plus importants porte-parole en matière de foresterie au Canada.

Notre mission consiste à faire progresser l'intendance des ressources forestières du Canada, d'assumer un leadership national en foresterie, de promouvoir la compétence parmi les professionnels de la foresterie, et chercher à sensibiliser le public sur les questions nationales et internationales en matière de foresterie.

Nos valeurs et nos convictions Nous croyons que les membres de I'Institut forestier du Canada/Canadian Institute of Forestry doivent avoir une connaissance approfondie des forêts du Canada. Nous croyons que la gestion de nos forêts en vue d'assurer le maintien de l'intégrité et de la dynamique des écosystèmes dans le cadre d'une perspective sociale acceptable doit s'inspirer d'une compréhension des principes de l'écologie, d'inventaires forestiers fiables et d'un contrôle suivi des travaux d'aménagement. Armés de cette compréhension, les membres de l'Institut se doivent de promouvoir l'utilisation rationnelle des forêts du Canada pour qu'elles demeurent la source inépuisable d'une vaste gamme de produits, et ce tout en conciliant les valeurs qui leur sont associées sur les plans économique, spirituel et récréatif, et de l'habitat faunique. Une large fourchette d'options s'offre à nous, depuis les forêts naturelles jusqu'aux fermes forestières soumises à des régimes d'aménagement intensif. Nous croyons que l'éventail des utilisations de la forêt en vue de répondre aux besoins des Canadiens et Canadiennes doit résulter d'un dialogue éclai ré entre les parties intéressées. Nous croyons aussi que les membres de l'IFC/CIF se doivent de jouer un rôle de premier plan pour faciliter ce dialogue.

\section{CIF/ IFC Executive Committee/ Comité exécutif}

President/ Président - Michael M cLaughlan

$1^{\text {st }}$ Vice-President/ $1^{\text {er }}$ vice-président - Chris Lee, R.P.F.

Past President/ Président sortant - Richard L. Macnaughton, R.P.F.

$2^{\text {nd }}$ Vice-President/ $2^{\text {ème }}$ vice-président - Doug Stables, R.P.F.

Executive Director/ directrice exécutive - RoxanneComeau, R.P.F.

\begin{tabular}{|c|c|}
\hline ctions & $\begin{array}{l}\text { Directors/ } \\
\text { directeurs }\end{array}$ \\
\hline uin & Don Willis \\
\hline & Ed Morrice \\
\hline 00 Chilcotin & Keith Dufres \\
\hline al Ontario & Peter Newtor \\
\hline hamplain & - \\
\hline londike & Susan Skaali \\
\hline ke of the Woods & 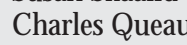 \\
\hline toba & Julie Ringas \\
\hline time & Doug Prosse \\
\hline oundland & Wayne Kelly \\
\hline ern Ontario & Wally Bidwe \\
\hline estern Ontario & Don Ni \\
\hline
\end{tabular}

Section Chairs/ président de section

Allan Stinson

$-$

Robert Currell

Gregory Cowman

Trevor Stanley

Daniel Rogers

Nikki Wood

Avery Dorland
Sections

Nova Scotia

Okanagan

Orleans

Ottawa Valley

Pacific

Rocky Mountain

Saskatchewan

Skeena

Southern Ontario

Vancouver

Vancouver Island
Directors/

directeurs

Mac Barkhouse

Rod Willis

Evelyne Thiffault

Jeff Young

Todd Nash

Darryl Sande

Bruce Ferguson

Mike Prueter

Charlie Klasen
Section Chairs/ président de section

Tony Bull

Geoff Clarke -

$-$

Brian Titus 